\title{
Radial velocities and axial rotation for a sample of chemically peculiar stars ${ }^{\star}$
}

\author{
H. Levato ${ }^{1, \star \star}$, S. Malaroda ${ }^{1, \star \star \star}$, N. Morrell ${ }^{2, \dagger}{ }^{\dagger}$, G. Solivella ${ }^{2}$ and M. Grosso ${ }^{1}$ \\ 1 Complejo Astronómico El Leoncito, Casilla de Correo 467, 5400 San Juan, Argentina \\ internet: levato@castec.edu.ar \\ 2 Facultad de Ciencias Astronómicas y Geofísicas, UNLP, Paseo del Bosque s/n, 1900, La Plata, Prov. de Bs.As. \\ Argentina
}

Received September 5, 1995; accepted January 4, 1996

\begin{abstract}
As part of a systematic project we have determined radial velocities and projected rotational velocities for a sample of 186 chemically peculiar stars which have been observed by the Hipparcos' satellite. The purpose is to provide necessary data to study the space velocities of peculiar stars.
\end{abstract}

Key words: stars: chemically peculiar — stars: kinematics — stars: rotation

\section{Introduction}

The Hipparcos' satellite has observed more than 100,000 stars with the purpose of measuring their proper motions with an accuracy never reached with observations from the ground. Also the space observations will provide trigonometric parallaxes for many of the objects. Kinematical studies will need the knowledge of the radial velocity of the stars observed by the satellite. In this paper we present the first results of a program started in 1989, at the national observing facility in Argentina, to provide radial velocities for samples of stars that have been targets of the Hipparcos' satellite. The first sample selected includes 186 chemically peculiar stars mostly of the CP2 class ( $\mathrm{Si}$, $\mathrm{Cr}, \mathrm{Sr}, \mathrm{Eu})(180)$ and a few of the CP4 class (He weak) (6) as defined by Preston (1974).

The space velocities and kinematical properties of the Ap stars have been studied previously with small sam-

Send offprint requests to: $\mathrm{H}$. Levato

${ }^{\star}$ Tables 1 and 2 are available in electronic form at the CDS via anonymous ftp 130.79.128.5

${ }^{\star \star}$ Member of the Consejo Nacional de Investigaciones Científicas y Técnicas de la República Argentina

${ }^{\star \star \star}$ Member of the Carrera del Investigador de la Comisión de Investigaciones Científicas de la Provincia de Buenos Aires

${ }^{\dagger}$ Member of the Consejo Nacional de Investigaciones Científicas y Técnicas de la República Argentina

${ }^{\ddagger}$ Visiting astronomer, Complejo Astronómico El Leoncito, which is operated under agreement between the Consejo Nacional de Investigaciones Científicas y Técnicas (CONICET) and the National Universities of La Plata, Córdoba and San Juan ples and less accurate data. Mouchet (1966) computed the space velocities for 227 Ap stars. Martinet (1966) using a sample of 101 stars, distinguished three groups from the appearance of the distribution of the projection of the space velocities in the galactic plane while Megessier (1974) using a sample of 21 stars found that the Ap (Si) stars behave similarly as normal B5 V to B8 V stars according with their kinematical properties and the Ap (Sr$\mathrm{Cr}-\mathrm{Eu}$ ) stars behave similarly as normal B9 V to A3 V.

\section{Observations and reduction}

We have obtained an average of 3 spectra per star for a sample of 180 CP2 stars and 6 CP4 stars observed by Hipparcos. The stars were selected from those in the south and brighter than 8 th magnitude and included in the Hipparcos Input Catalogue (HIC) (Turon et al. 1992). The spectra were secured with the $2.1 \mathrm{~m}$ telescope at CASLEO using a Boller \& Chivens cassegrain spectrograph, equipped with an EEV 8603 CCD of $385 \times 578$ pixels, each one of $22 \mu$ by $22 \mu$, and cooled with liquid nitrogen. The chip has a coating to increase the efficiency in the UV. The 2 pix resolution of the spectra is $1.3 \AA$ and the wavelength range recorded on the chip is a little less than $400 \AA$. We centered the spectrum on $\mathrm{H} \gamma$. We have also, for several of the program stars, one spectrum per object taken on photographic plates as described below.

Radial velocity standard stars from the IAU list and from the list provided by Feckel (1985) were observed with the same equipment. We have also obtained under the same conditions a good number of $v \sin i$ standards from 
Slettebak's list (Slettebak et al. 1975). We did not provide an independent spectral classification for the stars in our sample due to the small spectral range recorded on the CCD detector. However as has been said above, for most of the stars observed we have in our plate files at least one spectrum taken with the same spectrograph using the photographic plate as detector. This material was taken for radial velocity measurements (dispersion $29 \AA \mathrm{mm}^{-1}$ ) so it is not adequate for spectral classification. We used these spectrograms to verify the peculiar characteristics of the spectra of the program stars. We have indicated in the last column of Table 2 the spectral classification quoted in the Catalogue of Ap and Am Stars (Renson et al. 1991) and between brackets any discrepancy of the peculiarity that we have noted on our plates. An asterisk before the spectral classification means that no photographic plate is available and only the types quoted in Renson et al. (1991) are included in the Table. The reduction of the digital data was made with $\mathrm{IRAF}^{1}$ version 2.10 . The task splot was used for the determination of the wavelengths of the lines used for measuring the radial velocities. The same task was used for measuring the fwhm of Mg II $\lambda$ 4481 and He I $\lambda 4471$ which are the lines measured for deriving the $v \sin i$ values (see Valdes 1992).

\section{Results and discussion}

\subsection{Radial velocities}

Radial velocities were derived measuring the following individual lines when possible: Ca I $\lambda$ 4226, Fe II $\lambda$ 4233, $\mathrm{H} \mathrm{I} \mathrm{H} \gamma$, Fe I $\lambda \lambda 4405,4415,4383$, He I $\lambda 4471$, and $\mathrm{Mg}$ II $\lambda$ 4481. We used the $d$ option in the splot task for the fitting of the gaussian profiles and the computation of the position of the line.

The results of the measuring process are presented in Table 1 (available in electronic form only) where we have indicated, for the 186 stars, the HD number, the Julian Date and the heliocentric radial velocity for each measured spectrum jointly with its probable error deduced from the dispersion of the values given by the various lines, and the number of lines included in the average.

\subsection{Axial rotational velocities}

The axial rotational velocities were determined through the relation between the fwhm of the lines He I $\lambda 4471$ and $\mathrm{Mg}$ II $\lambda 4481$ and the $v \sin i$ values for a group of standard stars taken from Slettebak et al. (1975). For the program stars we have measured the fwhm for the same lines and using the calibration curves determined with the standard stars we have calculated the $v \sin i$ values. Figure 1 shows one of the calibration curves. In those cases in which both lines could be measured we have averaged

\footnotetext{
${ }^{1}$ Distributed by NOAO, Kitt Peak National Observatory, operated by AURA,Inc. under agreement with the NSF.
}

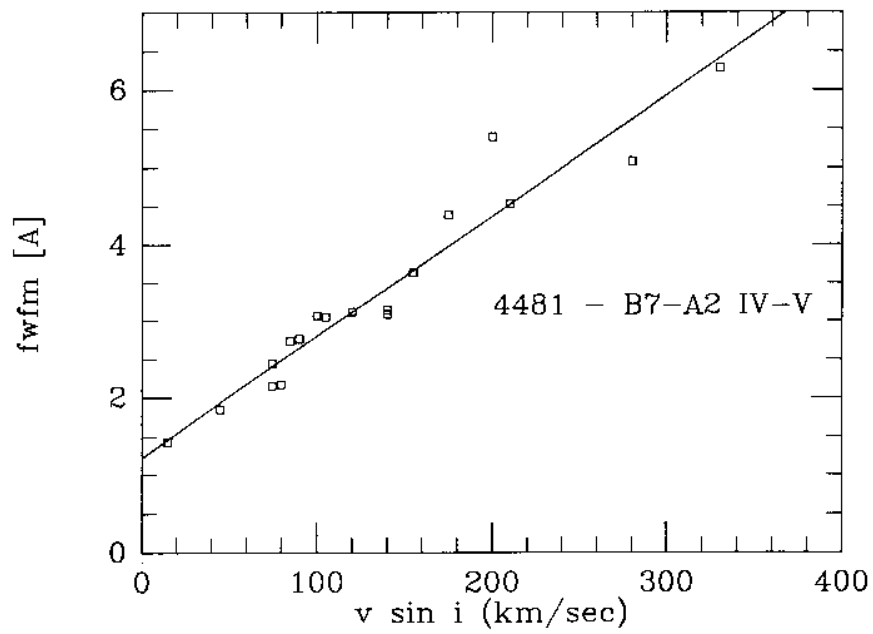

Fig. 1. $v \sin i$ vs. fwhm of MgII $\lambda 4481$ calibration for the spectral range $\mathrm{B} 7-\mathrm{A} 2$

the resulting values. The final results $(v \sin i)$ are presented in Table 2 where we have indicated in successive columns: (1) the HD number, (2) the average heliocentric radial velocity for each program star, (3) the probable error of the average, (4) the number of spectra included in the average, (5) the external probable error (see Conti et al. 1977), (6) the internal probable error (see Popper 1974), (7) the quotient between the external and internal errors, (8) the probability that the observed sample of radial velocity measurements for each star was drawn from a random population of measurements as explained below, (9) the $v \sin i$ value, and (10) the spectral classification according to the explanation given at the end of Sect. 2 .

The purpose of this paper is to publish the data but let us make some statistics with them to check the consistency of the results. There are $162 \mathrm{CP} 2$ stars with values of $v \sin i$ determined in our sample of Table 2. The average projected axial rotation for this sample is $47 \pm 3 \mathrm{~km}$ $\mathrm{s}^{-1}$ (s.e. of the mean) with $\sigma$ (s.e.) $=36 \mathrm{~km} \mathrm{~s}^{-1}$. We have taken $15 \mathrm{~km} \mathrm{~s}^{-1}$ as the $v \sin i$ value for all stars with $v$ $\sin i \leq 30 \mathrm{~km} \mathrm{~s}^{-1}$ in order to compute this average. The latter is a little larger than the average $v \sin i$ for the 102 CP2 stars brighter than 6.5 mag. from the Bright Star Catalogue, (Hoffleit \& Jaschek 1982) which have been recently measured by Abt \& Morrell (1995). Their value is $32 \pm 2 \mathrm{~km} \mathrm{~s}^{-1}$ (s.e of the mean) with a dispersion of $24 \mathrm{~km}$ $\mathrm{s}^{-1}$. The difference, is understandable because with our spectra we cannot resolve more than $30 \mathrm{~km} \mathrm{~s}^{-1}$ in the $v$ $\sin i$ values, while Abt \& Morrell (1995) could do better with $10 \mathrm{~km} \mathrm{~s}^{-1}$ resolution. We have not noticed statistically significant differences in the average $v \sin i$ values between the Si stars and the Sr-Cr-Eu stars.

From the 180 CP2 stars in Table 2, 136 have three spectra or more. For each of them, we have indicated in the same table the probability $(\mathrm{P})$, expressed as a percentage, that 
the observed radial velocity sample was drawn from a random population. An analysis of variance test (Conti et al. 1977) for this sample of 136 stars, indicates that $28 \%$ of the objects will have a variable radial velocity. We consider as variable, all stars whose radial velocity measurements have a probability lower than $0.5 \%$ of being drawn from a random population. This value of the significance level listed for each star in Table 2 was derived empirically from our own data.

Figure 2 shows the distribution of the quotient $E / I$ for the $170 \mathrm{CP} 2$ stars with 2 or more observations. Among this sample, $72 \%$ of the stars have $E / I$ values lower than 3.0. Seventeen stars have $E / I$ values falling in the range $3.0 \leqq E / I \leqq 4.0$. We have assumed that the stars with $E / I$ values larger than 3.5 are radial velocity variables. With this assumption we have found that $20 \%$ may be radial velocity variables. These numbers agree with previous determinations of the incidence of spectroscopic binaries among Ap stars (Abt \& Snowden 1973).

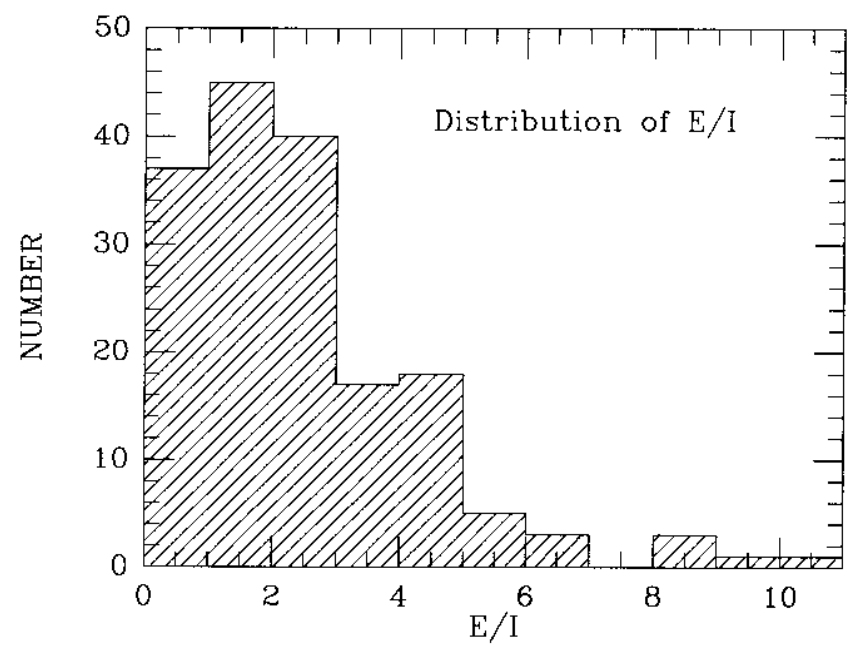

Fig. 2. Distribution of $E / I$ for the $170 \mathrm{CP} 2$ stars

Summarizing, we have presented radial velocities for a sample of $186 \mathrm{CP}$ stars observed by Hipparcos, mostly of the CP2 class, and we have checked the consistency of these new data and previous statistical results. We have concluded that the incidence of radial velocity variables among CP2 stars is lower than among late B and early A type stars in agreement with previous analysis, and that the average $v \sin i$ for the CP2 stars in our program agrees also with previous determinations.

Acknowledgements. We appreciate very much the help of the technical staff at the Complejo Astronómico El Leoncito during the observations and the reduction of the data. We also thanks the support from the Consejo Nacional de Investigaciones Científicas y Técnicas through grants for travelling. We appreciate also the contribution of the referee, Dr. P. Renson, to the improvement of the final presentation of the paper.

\section{References}

Abt H.A., Morrell N., 1995, ApJS 99, 135

Abt H.A., Snowden M.S., 1973, ApJS 25, 137

Andersen J., Nordstrom B., 1983, A\&AS 52, 471

Batten A.H., Fletcher J.M., MacCarthy D.G., 1989, Eighth Catalogue of the Orbital Elements of Spectroscopic Binary Systems, Pub. D.A.O. XVII, 1

Conti P.S., Garmany C., Hutchings J., 1977, ApJ 215, 561

Donoyelle J., 1987, A\&AS 70, 373

Dworetsky M.M., 1982, Obs. 102, 138

Feckel F.C., 1985, in: Stellar Radial Velocities, IAU Colloq. 88. In: Davis Philip A.G. and Latham D.W. (eds.). L. Davis Press Inc., Schenectady, New york, U.S.A., p. 335

Fisher W.A., 1987, MNRAS 224, 393

Hoffleit D., Jaschek C., 1982, The Bright Star Catalogue, 4th rev. ed.: New Haven: Yale Univ. Observatory

Levato H., Malaroda S., Morrell N., Solivella G., 1987, ApJS 64,487

Martinet L., 1966, Bull. Astron. Ser. 3, 95

Mathys G., 1990, A\&A 232, 151

Mc Fadzean A.D., Hilditch R.W., Hill G., Aikman C.L., Mégessier C., 1974, A\&A 37, 439

Mouchet M.T., 1966, Bull. Astron. Ser. 3, 87

Nordstrom B., Andersen J., 1985, A\&AS 61, 53

Perry C.L., Hill G., Younger P.F., Barnes J.V., 1990, A\&AS 86,415

Popper D.M., 1974, AJ 79, 1307

Preston G.W., 1974, ARA\&A 12, 257

Renson P., Gerbaldi M., Catalano F.A., 1991, A\&AS 89, 429

Slettebak A., Collins G.W., Boyce P.B., White N.M., Parkinson T.D., 1975, ApJS 29, 137

Turon C., Crézé W., Egret D., et al., 1992, The Hipparcos Input Catalogue, ESA-SP 1136, Noordwijk, The Netherlands

Valdes F., 1992, ASP Conf. Ser. Astron. Data Anal. Soft. Syst. I, 417

Zentelis N., 1983, A\&AS 53, 445 
Table 2. Statistical results

\begin{tabular}{|c|c|c|c|c|c|c|c|c|c|}
\hline HI) & $\langle\mathbf{V R}\rangle$ & pe & $n$ & lixt.pe & Int.pe & $\mathbf{E} / \mathbf{I}$ & $p \%$ & $v \sin \mathrm{i}$ & Spectral Type \\
\hline $35 \times(1$ & +12.0 & 5.4 & (4) & 10.8 & 3.8 & 2.8 & 0.5 & 90 & $\mathrm{Si}$ \\
\hline 6783 & +5.6 & 1.3 & (3) & 2.3 & 4.6 & 0.5 & $>25$ & $<31)$ & * Bs8 Si \\
\hline 878.3 & -4.3 & 12.8 & (3) & 22.2 & 3.4 & 6.5 & $<0.1$ & $<31$ & $\Lambda 2$ SrCrEu \\
\hline 10840 & 19.4 & 2.1 & (6) & 5.1 & 2.1 & 2.4 & 1 & $<30$ & I39 Si (SiCr:) \\
\hline 22488 & 15.2 & 4.0 & (5) & 8.9 & 2.1 & 4.2 & $<1.1$ & 125 & A3 CrSrEu \\
\hline 23207 & 6.9 & 3.1 & (4) & 6.2 & 2.8 & 2.2 & $10-5$ & $<30$ & *A2 SrCr \\
\hline 24188 & 6.9 & 1.6 & (3) & 2.8 & 2.9 & 1.0 & $>25$ & $<30$ & $\Delta 0 \mathrm{Si}$ \\
\hline 24825 & 5.0 & 10.6 & (2) & 15.0 & 1.8 & 8.3 & - & - & B9 CrEu (CrSrEu) \\
\hline 27463 & 26.5 & 2.4 & (4) & 4.8 & 2.6 & 1.8 & $16-5$ & 40 & Al EuCr (SrCrEu) \\
\hline 28299 & 22.6 & 3.9 & (4) & 7.8 & 2.4 & 3.2 & 1 & $\langle 31)$ & IBS $\mathrm{Si}$ \\
\hline 29435 & 14.5 & 1.2 & (1) & - & - & - & - & - & I39 Si (normal spectrum) \\
\hline 31612 & 3.7 & 4.1 & (5) & 9.2 & 1.9 & 4.8 & $<0.1$ & $<30$ & I39 $\mathrm{Si}$ \\
\hline 31225 & 5.7 & 5.5 & (4) & 11.0 & 2.2 & 5.0 & $<0.1$ & $<30$ & A3 SrCrEu (SrCr:) \\
\hline 341061$)$ & 17.1 & 5.4 & (4) & 10.8 & 2.2 & 4.9 & $<0.1$ & 65 & 139 $\mathrm{SiCr}$ (SiCrSr) \\
\hline 34631 & 6.3 & 1.5 & (2) & 2.1 & $2.1)$ & 1.0 & - & $<30$ & $* 139 \mathrm{Si}$ \\
\hline 34797 & 17.3 & 2.0 & (2) & 2.9 & 0.6 & 4.8 & - & 30 & 138 He weak \\
\hline 36916 & 6.9 & 2.8 & (2) & 4.0 & 2.2 & 1.8 & $\cdot$ & 35 & 138 He weak \\
\hline 37808 & 17.9 & 1.3 & (3) & 2.2 & 1.7 & 1.3 & $>25$ & 45 & $139 \mathrm{Si}$ \\
\hline 38471 & 12.7 & 2.2 & (3) & 3.9 & 1.3 & $3.1)$ & $0.5-17.1$ & 30 & (39) $\mathrm{Si}$ \\
\hline 38719 & 11.3 & 1.8 & (2) & 2.6 & 1.6 & 1.6 & - & 50 & AO SrCrEu \\
\hline 39353 & 25.6 & 3.9 & (3) & 6.7 & 2.8 & 2.4 & 2.5 & 95 & $\mathrm{IBS} \mathrm{Si}$ \\
\hline 40071 & 13.1 & $\theta, 9$ & (1) & - & $\cdot$ & - & $\cdot$ & - & $139 \mathrm{~V} \mathrm{Si}$ \\
\hline 41089 & 16.1 & 1.3 & (2) & 1.9 & 2.2 & 0.9 & - & - & I39 SrCritu (Si) \\
\hline 42326 & 18.0 & 3.9 & (2) & 5.5 & 3.4 & 1.6 & - & 75 & A0 CrEu (CrSrEu) \\
\hline 42536 & 16.0 & 12.4 & (2) & 17.6 & 2.2 & 8.0 & - & 60 & A0 SrCr (SrCrEv:) \\
\hline 42695 & 1.5 & 5.1 & (3) & 8.8 & 2.4 & 2.2 & 0.5 & 70 & IB9 Si \\
\hline 44293 & 22.2 & 4.3 & (3) & 7.4 & 3.3 & 1.4 & 2.5 & $<30$ & $\mathrm{~A} t \mathrm{Cr}$ (SrSiCr) \\
\hline 44953 & 20.4 & 0.9 & (9) & 2.7 & 1.8 & 1.5 & 10 & $<\mathbf{M})$ & B8 He weak \\
\hline 45439 & 28.5 & 3.3 & (3) & $5 . x$ & 2.9 & 2.6 & 10 & 105 & By Si \\
\hline 46462 & 2.3 .3 & 2.8 & (3) & 4.8 & 2.9 & 1.6 & $25-10$ & $<30$ & I3y Si (B39 V) \\
\hline 47116 & 4.7 & 0.4 & (2) & 0.6 & 2.6 & 0.2 & - & $<30$ & $139 \mathrm{Si}$ \\
\hline 49333 & 15.1 & 1.1 & (8) & 3.0 & 1.7 & 1.8 & 2.5 & 60 & I39 Si (He weak) \\
\hline 52993 & 14,3 & 6.7 & (3) & 11.7 & 1.7 & 6.9 & $<0.1$ & 145 & $139 \mathrm{Si}$ \\
\hline 56273 & 9.9 & 0.1 & (2) & 1..2 & 2.9 & 0.1 & - & 60 & $\mathrm{I39} \mathrm{Si}$ \\
\hline 56354 & 22.4 & 1.5 & (3) & 2.6 & 2.0 & 1.3 & 25 & 40 & A0 EuCrSr \\
\hline 56455 & 27.6 & 1.7 & (3) & 3.9 & 1.7 & 2.3 & $5-25$ & - & An $\mathbf{S i}$ \\
\hline 56809 & -7.3 & 2.0 & (3) & 3.5 & 2.3 & 1.5 & $10-5$ & 80 & $139 \mathrm{SrCr}$ \\
\hline 569017 & 21.7 & 1.2 & (3) & 2.1 & 1.9 & 1.1 & $<\mathbf{2 5}$ & $<30$ & A0 $\mathrm{Si}$ \\
\hline 58292 & 12.6 & 0.2 & (3) & 0.3 & 2.2 & 0.2 & $>25$ & $<30$ & A0 $\mathbf{S i}$ \\
\hline 58448 & 20.0 & 4.9 & (3) & 8.4 & 2.3 & 3.6 & $0.5-4.1$ & 65 & BX Si \\
\hline 60559 & -2.8 & 12.0 & (3) & 20.8 & 2.4 & 8.9 & $<0.1$ & $<30$ & $\mathrm{~B} 8 \mathrm{Si}$ \\
\hline 61966 & -0.1 & 4.3 & (2) & 6.1 & 4.0 & 1.5 & - & 65 & IBX Si \\
\hline 62317 & 24.2 & 1.3 & (3) & 2.2 & 1.7 & 1.3 & $>25$ & $<30$ & $\mathrm{Al} \mathbf{S i}$ \\
\hline 62531$)$ & 6.8 & 4.4 & (1) & - & - & - & - & 60 & ${ }^{*} 139 \mathrm{LuCr}$ \\
\hline 62535 & 30.8 & 5.6 & (3) & 9.8 & 0.9 & 10.9 & $<0.1$ & $<30$ & A0 Si (SiCr:Sr:) \\
\hline 62553 & 13.5 & 6.4 & (3) & 11.1 & 2.7 & 4.1 & $<0.1$ & 55 & Al) $\mathrm{Sr}^{-}(\mathrm{SrCr})$ \\
\hline
\end{tabular}


Table 2. continued

\begin{tabular}{|c|c|c|c|c|c|c|c|c|c|}
\hline $\mathbf{H I D}$ & $\langle\mathbf{V R}\rangle$ & pe & n & lixt.pe & Int.pe & $\mathbf{b} / \mathbf{1}$ & $1 P \%$ & $v \sin i$ & Spectral Type \\
\hline 62556 & 23.8 & 3.4 & (3) & 5.9 & 3.2 & 1.8 & 10) & 35 & A0 $\mathrm{EuCr}(\mathrm{Cr} \mathrm{Sr})$ \\
\hline 62640 & 29.7 & 3.4 & (3) & 5.9 & 2.3 & 2.6 & $2-1$ & 45 & I39 Si \\
\hline 62714 & -7.2 & 2.4 & (12) & 7.0 & 2.3 & 3.1 & $<0.1$ & $<30$ & B8 He weak \\
\hline 63401 & 16.3 & 6.3 & (3) & 14.9 & 3.2 & 3.4 & 0.5 & 50 & I39 Si \\
\hline 64784 & 28.1 & 2.7 & (2) & 3.8 & 3.7 & 1.0 & - & 25 & $\mathrm{~B} 9 \mathrm{Si}$ \\
\hline 65836 & 9.1 & 2.2 & (3) & 3.8 & 4.9 & 0.8 & $>25$ & 30) & A2 SrCrliu \\
\hline 66605 & 23.4 & 4.2 & (2) & 6.0 & 2.1 & 2.9 & - & $\cdot$ & SiII, CrII, SrII A0 \\
\hline 66698 & 16.8 & 10.5 & (2) & 14.9 & 3.8 & 3.9 & - & $<\mathbf{3 4}$ & Al Eu (Sr Si:) \\
\hline 67835 & 18.1 & 2.9 & (3) & 5.1 & 3.2 & 1.6 & $25-10$ & 30 & I38 Si \\
\hline 68292 & 5.9 & 2.0 & (3) & 3.4 & 2.2 & 1.5 & $25 \cdot 10$ & $<30$ & $139 \mathrm{Si}$ \\
\hline 68561 & 15.1 & 5.2 & (3) & 9.1 & 2.0 & 4.5 & $<0 . \mathrm{I}$ & $<30$ & $139 \mathrm{Si}$ \\
\hline 70507 & 18.2 & 1.2 & (3) & 2.1 & 2.8 & 0.7 & $>25$ & $31)$ & B9 SiCr \\
\hline 71491 & 22.0 & 3.0 & (3) & 5.2 & 1.7 & 3.4 & $1-0.5$ & 40 & I38 Si \\
\hline 72303 & 10.1 & 4.0 & (3) & 6.9 & 2.2 & 3.1 & 1 & $<30$ & I39 $\mathrm{Si}(\mathrm{SiCrSr})$ \\
\hline 72634 & 6.8 & 1.7 & (3) & 2.9 & 1.5 & 1.9 & 2.5 & - & *AO CrEuSr \\
\hline 72881 & 13.7 & 0.7 & (3) & 1.3 & 3.2 & 0.4 & $>25$ & $<30$ & $139 \mathrm{Si}(\mathrm{SiCr})$ \\
\hline 72976 & 24.8 & 0.9 & (3) & 1.5 & 3.7 & 0.4 & $>25$ & $<30$ & $\mathrm{~A}) \mathrm{Si}$ \\
\hline 74168 & 2.4 & 6.4 & (3) & 11.0 & 3.9 & 2.8 & $2.5-1$ & 55 & B9 Si (SiSr) \\
\hline 74388 & 21.9 & 5.3 & (3) & 9.1 & 2.6 & 3.5 & 0.1 & 40 & I $38 \mathbf{S i}$ \\
\hline 74888 & 41.6 & 0.5 & (3) & 0.9 & 1.9 & 0.5 & $>25$ & 60 & I39 Si \\
\hline 75445 & 7.2 & 1.7 & $(4)$ & 3.0 & \$.7 & 4.3 & $25 \cdot 10$ & 95 & A3 SrEu \\
\hline 75989 & -18.1 & 4.3 & (3) & 7.5 & 2.5 & 3.0 & $<0.1$ & $<30$ & 139 Si (SiSrCr) \\
\hline 76614 & 6.6 & 1.3 & (3) & 2.3 & 3.6 & 0.6 & $>25$ & $31)$ & An $\mathbf{S i}$ \\
\hline 76897 & 23.4 & 0.9 & (3) & 1.6 & 2.4 & 0.7 & $>25$ & 35 & I3y Si (SiSr) \\
\hline 77653 & 20.3 & 1.9 & (4) & 3.8 & 2.5 & 1.5 & 10 & 45 & I39 Si (SiSr) \\
\hline 78568 & 18.0 & 4.3 & (3) & 7.5 & $3.1)$ & 2.5 & $2.5-1$ & 50 & $139 \mathrm{Si}$ \\
\hline 80282 & 23.5 & 3.3 & (4) & 6.5 & 2.2 & 2.9 & 1 & 45 & $\Lambda 0 \mathbf{S i}$ \\
\hline 810109 & 12.9 & 11.0 & (2) & 15.5 & 2.7 & 5.7 & - & 85 & A3 CrSrSi (SrCrFuSi) \\
\hline 81141 & -6.8 & 5.6 & (3) & 9.7 & 3.3 & 2.9 & 1 & 155 & I39 $\mathrm{Si}$ \\
\hline 82093 & -15.1 & 5.9 & (3) & 10.2 & 2.6 & 3.9 & $5-2.5$ & 45 & $\triangle 2 \mathrm{SrCrFu}(\mathrm{SrCr})$ \\
\hline 82567 & 12.8 & 4.0 & (3) & 6.9 & 3.2 & 2.2 & $\$ 1.1$ & $<30$ & $\mathrm{IB9} \mathrm{Si}$ \\
\hline 83368 & -9.3 & 2.11 & (3) & 3.5 & 2.8 & 1.2 & 25 & 110 & As SrCrliu \\
\hline 83625 & 12.5 & 5.3 & (3) & 9.2 & 2.3 & 4.0 & $0.5-0.1$ & 70 & An SiSr \\
\hline 85892 & 17.5 & 11.1 & (2) & 15.7 & 2.9 & 5.4 & + & 45 & $* \mathbf{B S 8} \mathrm{Si}$ \\
\hline 86199 & 7.1 & 0.3 & (2) & 0.4 & 7.11 & 0.1 & - & $<39$ & By Si (SiCr) \\
\hline 86216 & 10.9 & 15.9 & $(2)$ & 22.5 & 5.2 & 4.3 & $\cdot$ & 420 & I39 He weak \\
\hline 87653 & 5.8 & 5.1 & (1) & $\cdot$ & $\cdot$ & - & - & $\cdot$ & *I39 Si \\
\hline 88158 & 16.4 & 0.9 & (3) & 1.6 & 1.6 & 1.0 & $>25$ & 60 & I38 Si (SiCrSr) \\
\hline 89103 & 2.0 & 1.5 & (3) & 2.6 & 3.1 & 0.8 & $>25$ & $<30$ & 139) Si (SiSrer) \\
\hline 89192 & -2.7 & 4.2 & (3) & 7.3 & 3.4 & 2.1 & 5 & 80 & AO CrSrEu \\
\hline $9(\mathrm{k}) 44$ & 22.9 & 2.7 & (4) & 5.4 & 2.4 & 2.2 & 0.5 & 30 & $139 \mathrm{SiCrSr}$ (SiCrSrEu) \\
\hline 90763 & -20.5 & 1.7 & (3) & 2.9 & 1.9 & 1.5 & $25-10$ & 65 & $\mathrm{Al} \mathrm{Sr}(\mathrm{SrCr})$ \\
\hline 91089 & -3.5 & 3.6 & (3) & 6.2 & 3.3 & 1.9 & 16 & $<30$ & 139 $\mathrm{Si}$ \\
\hline 91239 & 23.4 & 2.5 & (3) & 4.3 & 2.4 & 1.8 & 25 & $<31$ & A6 FuCrSi (SrCrSi) \\
\hline 91590 & -0.1 & 4.8 & (3) & 8.3 & $2.1)$ & 4.1 & 0.1 & $<30$ & I39 Si \\
\hline 92106 & -27.7 & 8.1 & (3) & 14.1 & 2.2 & 6.4 & all.1 & $<30$ & A0 CrSrEu (SrCrlin:) \\
\hline 92.379 & 1.1 & 12.6 & (3) & 21.8 & 2.4 & 9.1 & $<0.1$ & 811 & I3\$ Si (Si-Sr?) \\
\hline 93821 & -10.2 & 7.7 & (2) & 11.0 & 2.7 & 4.1 & - & $<30$ & I39 Si (SiCr:Sr:) \\
\hline 94660 & 2.3 .4 & 2.3 & (3) & 4.0 & 2.1 & 1.9 & $10-5$ & $<\mathbf{3 1 1}$ & AO LuCrSr (SiCrSrEu) \\
\hline
\end{tabular}


Table 2. continued

\begin{tabular}{|c|c|c|c|c|c|c|c|c|c|}
\hline HI) & $\langle\mathrm{VR}\rangle$ & pe & n & Ext.pe & lnt.pe & $\mathbf{E} / \mathbf{I}$ & $\mathbf{p} \%$ & v sin i & Spectral Type \\
\hline 95198 & 13.8 & 6.4 & (3) & 0.7 & 3.1 & 0.2 & $>25$ & 45 & I39 Si \\
\hline 96451 & -26.4 & 3.1 & (4) & 6.2 & 3.1 & 2.0 & $5-2.5$ & 80 & $\Lambda 0 \mathrm{Sr}$ \\
\hline 96910 & 1.8 & 2.7 & (1) & - & - & - & - & 40 & *139 SiCrEu \\
\hline 97986 & -3.2 & 2.9 & (3) & 5.11 & 2.3 & 2.2 & $10-5$ & $<30$ & BS Si (SiCr:) \\
\hline 98340 & 3.6 & 1.4 & (3) & 2.5 & 1.8 & 1.4 & 25 & 110 & $139 \mathrm{Si}(\mathrm{CrSi})$ \\
\hline 98457 & 11.9 & 5.0 & (3) & 8.7 & 1.9 & 4.6 & $<0.1$ & $<30$ & $\Lambda 0 \mathrm{Si}$ \\
\hline 101410 & 1.9 & 3.5 & (3) & 6.1 & 2.6 & 2.3 & 2.5 & 85 & $* A 0 \mathrm{liuCr}$ \\
\hline 103962 & -27.5 & 2.6 & (5) & 5.7 & 2.5 & 2.3 & 1 & - & $139 \mathrm{Si}$ (normal spectrum) \\
\hline 104810 & -20.5 & $2.1)$ & (4) & 4.0 & 1.7 & 2.3 & 10 & 120 & $138 \mathrm{Si}$ \\
\hline 105770 & -13.1 & 10.7 & (2) & 15.2 & 3.5 & 4.3 & $\cdot$ & $<30$ & I3y Si \\
\hline 105999 & $-\mathbf{- 1 . 1}$ & 6.1 & (3) & 10.5 & 3.9 & 2.7 & $2.5-1$ & $\cdot$ & Il SrCr \\
\hline $11238 \mathrm{I}$ & 5.4 & 1.3 & (3) & 2.3 & 2.3 & 1.0 & $>25$ & - & A0 SiCr (SiCrSr) \\
\hline 114,365 & 2.9 & 4.1 & (4) & 8.2 & 2.0 & 4.1 & $<0.1$ & $<30$ & $\mathbf{A O} \mathrm{Si}$ \\
\hline 116114 & 3.6 & 3.5 & (3) & 6.0 & 2.0 & 3.0 & 6.5 & 65 & FO SrEuCr \\
\hline 116458 & -5.3 & 3.7 & (3) & 6.4 & 2.1 & 3.1 & $<0.1$ & $<30$ & A0 SiEuCr (CrSrEu?)) \\
\hline 118242 & -4.1 & 7.1 & (2) & 10.1 & 2.6 & 3.9 & - & 93 & B9 Si (normal spectrum) \\
\hline 118473 & -20.0 & 0.9 & (3) & 2.7 & 3.1 & 0.9 & $>25$ & 150 & I39 Si (SiCr:) \\
\hline 118816 & .35 .9 & 1.6 & (3) & 2.7 & 3.0 & 0.9 & $>25$ & $<30$ & B9 Si (Si:) \\
\hline 118913 & -4.8 & 1.7 & (3) & 3.4 & 3.6 & 0.9 & $>25$ & 40 & *A0 EuCrSr \\
\hline 119308 & -4.6 & 1.2 & (3) & 2.1 & 2.9 & 0.7 & $>25$ & 40 & I39 SrCrẼ (SrCr) \\
\hline 122208 & 8.2 & (1.6 & (3) & 0.9 & 3.2 & 0.3 & $>25$ & 105 & $* \wedge 2$ SrCrEu \\
\hline 123112 & -8.4 & 9.8 & (4) & 19.6 & 4.1 & 4.8 & $<0.1$ & 205 & A0) $\mathrm{SiCr}(\mathrm{Al} V)$ \\
\hline 125630 & -9.2 & 5.8 & (3) & 10.1 & 3.8 & 2.7 & 1 & 45 & A2 SiCrSr (CrSrEu:) \\
\hline 127453 & 11.3 & 1.1 & (3) & 2.0 & 2.5 & 0.8 & $>25$ & 30 & $* 138 \mathrm{Si}$ \\
\hline 127575 & 7.0 & 2.9 & (2) & 4.0 & 3.2 & 1.2 & - & $<30$ & *139 Si \\
\hline 128775 & 5.6 & 4,0 & (3) & 6.9 & 2.7 & 2.6 & 2.5 & $<311$ & 139 Si (SiCir:) \\
\hline 128974 & -24.4 & 3.2 & (4) & 6.3 & 2.6 & 2.4 & 1 & 60 & Al) Si (Si:) \\
\hline 129750 & 4.5 & 2.9 & (3) & 5.0 & 4.4 & 1.1 & $25-10$ & $<30$ & $* 139 \mathrm{Si}$ \\
\hline 129899 & 2.5 & 6.4 & (2) & 0.6 & 2.0 & 0.3 & - & 40 & ${ }^{*} \mathrm{~A} 0 \mathrm{Si}$ \\
\hline 130335 & -15.2 & 1.0 & (2) & 1.4 & 3.4 & 0.4 & - & $<30$ & $\mathbf{A 2} \mathbf{S j}$ \\
\hline 132322 & -13.3 & 2.4 & (2) & 3.3 & 3.5 & (1.9) & - & 85 & $\Lambda 7$ SrCrEu \\
\hline 133652 & 2.5 & 2.9 & (3) & 4.9 & 4.9 & 1.0 & $>25$ & 40 & 139 Si (SiCr:) \\
\hline 133792 & 6.5 & 1.2 & (4) & 2.3 & 2.6 & 10.9 & $>25$ & $<30$ & Al $\mathrm{SrCr}$ \\
\hline 134214 & -7.7 & 4.9 & (3) & 8.5 & 3.5 & 2.4 & 2.5 .1 & - & *F2 SrCrEu \\
\hline 134874 & -29.0 & 5.8 & (3) & 10.1 & 3.3 & 3.1 & 0.5 & $<31$ & B9 Si (Si:) \\
\hline 135415 & -21.4 & 4.2 & (2) & 6.0 & 4.2 & 1.4 & - & $<30$ & B8 Si \\
\hline 137193 & -18.0 & 2.2 & (2) & 3.1 & 2.8 & 1.1 & - & 30 & * $\mathbf{3 9} \mathrm{Si}$ \\
\hline 137607 & 5.6 & 4.3 & (3) & 7.4 & 3.2 & 2.3 & $2.5-1$ & 40 & ${ }^{*} \mathbf{A} \mathbf{1} \mathbf{S i}$ \\
\hline 138497 & .40 .0 & 4.8 & (3) & 8.4 & 2.8 & $3 . \%$ & $0.5-1$ & 75 & 139 SrCrliu \\
\hline 138758 & 2.2 & 0.5 & (3) & 0.8 & 3.4 & 0.2 & 25 & 50 & $139 \mathrm{Si}(\mathrm{A} 0 \mathrm{~V})$ \\
\hline 138773 & -4.7 & 0.4 & (2) & 0.6 & 3.0 & 0.2 & - & 50 & *AO Si \\
\hline 139525 & -27.3 & 0.0 .3 & (2) & 0.1 & 2.3 & 0.04 & . & $<30$ & I38 Si \\
\hline 143473 & 0.3 & 3.0 & (4) & 6.1 & 3.0 & 2.0 & 1 & 30 & $139 \mathrm{Si}(\mathrm{SiCr})$ \\
\hline 144231 & -9.2 & 4.9 & (3) & 8.5 & 3.0 & 2.8 & 0.5 & $<311$ & I3) $\mathrm{Si}$ \\
\hline 145102 & -2.8 & 3.5 & (3) & 6.1 & 4.3 & 1.4 & $25.11)$ & 90 & I39 Si \\
\hline 147890 & -28.5 & 3.3 & (2) & 4.7 & 4.4 & 1.1 & - & 45 & *Ail SiSr \\
\hline 148199 & -28.1 & 3.7 & (3) & 6.3 & 2.9 & 2.2 & 2.5 & 40 & B9 SiSr (SiCr:) \\
\hline 149764 & 13.0 & 5.8 & (2) & 8.2 & 4.5 & 1.8 & - & 70 & ${ }^{*} \mathbf{\Lambda} \mathbf{I} \mathbf{S i}$ \\
\hline 150486 & -36.4 & 3.1 & (3) & 5.4 & 2.7 & 2.0 & 10 & 55 & B9 Si \\
\hline
\end{tabular}


Table 2. continued

\begin{tabular}{|c|c|c|c|c|c|c|c|c|c|}
\hline HI) & $\langle$ VR $\rangle$ & pe & n & Ext.pe & Int.pe & $\mathbf{E} / \mathbf{I}$ & $1 \%$ & $y \sin i$ & Spectral Type \\
\hline 150500 & -2.5 & 3.7 & (3) & 6.4 & 2.4 & 2.7 & 1 & 75 & $139 \mathrm{Si}$ \\
\hline 150549 & -2.6 & 3.6 & (3) & 6.2 & 4.1 & 1.5 & 25 & 75 & $\Delta 0 \mathrm{Si}$ \\
\hline 151363 & -10.9 & 1.0 & (3) & 1.7 & 4.4 & 0.4 & $>25$ & 105 & *B9 Si \\
\hline 151965 & -2.9 & 0.1 & (2) & 0.1 & 2.9 & 0.013 & - & 105 & B9 Si (SiSr:) \\
\hline 152273 & -3.1 & 9.2 & (3) & 16.0 & 3.3 & 4.8 & $<0.1$ & 160 & $\mathrm{~A}_{0} \mathrm{Si}(\mathrm{A0} \mathrm{V})$ \\
\hline 152564 & -6.0 & 4.5 & (3) & 7.7 & 4.3 & 1.8 & 10 & 75 & $\mathrm{~A} \mathbf{0} \mathrm{Si}$ \\
\hline 153201 & 0.2 & 1.6 & (3) & 2.7 & 2.1 & 1.3 & 25 & 45 & By Si (SiCr:) \\
\hline 155778 & -21.9 & 1.7 & (3) & 2.9 & 2.2 & 1.3 & $>25$ & 65 & $*$ IB8 Si \\
\hline 156853 & -24.0 & 1.7 & (3) & 5.9 & 4.3 & 1.4 & 25 & 41) & I38 Si \\
\hline 156869 & -29.3 & 2.5 & (4) & 4.9 & 2.7 & 1.8 & 16 & 51) & *A1 SrCrEu \\
\hline 157678 & 20.6 & 3.2 & (2) & 4.5 & 6.4 & 0.7 & - & 130 & ${ }^{*} \mathbf{B 9} 9 \mathrm{Si}$ \\
\hline 157751 & -15.7 & 0.6 & (1) & - & - & - & - & 30 & *B9 SiCr \\
\hline 158128 & 4.6 & 1.9 & (1) & - & - & - & - & 35 & ${ }^{*} \mathbf{B} 9 \mathrm{Si}$ \\
\hline 158175 & -12.2 & 6.0 & (2) & 8.5 & 2.8 & 3.0 & - & $<30$ & B8 Si (SiCrSr:) \\
\hline 158819 & -17.0 & 3.2 & (1) & - & - & $\cdot$ & $\cdot$ & - & J39 Si (B39/A0 normal) \\
\hline 159286 & 25.6 & 3.3 & (1) & $\cdot$ & $\cdot$ & - & - & 100 & ${ }^{*} \mathrm{B9} 9 \mathrm{SiCr}$ \\
\hline 159846 & -7.2 & 0.4 & (2) & 0.6 & 2.6 & 0.2 & - & $<30$ & I39 Si (SiCr) \\
\hline $160+468$ & 41).4 & $3.1)$ & (1) & - & $\cdot$ & - & - & 100 & $* \mathrm{l} 2 \mathrm{Sr}-\mathrm{Cr}$ \\
\hline 161277 & -2.3 .8 & 4.1 & (2) & 5.8 & 1.7 & 3.4 & - & $<30$ & I39 Si (SiSr) \\
\hline 166427 & -25.5 & 6.7 & (3) & 1.3 & 2.9 & 0.4 & $>25$ & 35 & $139 \mathrm{SiCr}$ \\
\hline 166953 & 1.1 & 2.4 & (4) & 4.9 & 3.1 & 1.6 & $25-10$ & 30 & $\Delta \mathbf{0} \mathrm{Si}$ \\
\hline 169594 & -1.3 & 2.7 & (3) & 4.7 & 5.2 & 0.9 & $>25$ & 60 & A2 Si (CrSrSi) \\
\hline 172690 & -2.3 & 3.6 & (3) & 6.3 & 2.8 & 2.2 & 25 & $<30$ & ${ }^{*} \Lambda 0 \mathrm{SiCrSr}$ \\
\hline 174779 & $\mathbf{3 . 3}$ & 2.0 & (4) & 4.0 & 2.7 & 1.5 & $<25$ & 45 & AO Si (SiCrSr) \\
\hline 176196 & 3.0 & 6.5 & (3) & 11.3 & 2.8 & 4.0 & $<0.1$ & $<30$ & *139 liuCr \\
\hline 181018 & 1.6 & 3.6 & (4) & 7.3 & 2.6 & 2.8 & 1 & $<30$ & B9 Si (CrSr:EuSi) \\
\hline 185183 & -16.1 & 5.1 & (4) & I0.1 & 2.1 & 4.8 & $<0.1$ & 50 & $\mathbf{B 9} \mathrm{Si}$ \\
\hline 186117 & -18.8 & 8.2 & (3) & 14.3 & 2.7 & 5.3 & $<0.1$ & $<30$ & *AO SrCrEu \\
\hline 187473 & -1.3 & 7.0 & (3) & 12.1 & 2.1 & 5.8 & $<0.1$ & $<30$ & 139 SrEuSi \\
\hline 191507 & -7.3 & 8.2 & (3) & 14.2 & 4.9 & 2.9 & $0.5-1) .1$ & 125 & I39 Si \\
\hline 191796 & -6.9 & 2.4 & (3) & 4.2 & 5.3 & 0.8 & $>25$ & 75 & A1) EuCr (CrEuSr) \\
\hline 192674 & 2.4 & 2.11 & (3) & 3.4 & 4.8 & 0.7 & $>25$ & 55 & I39 $\mathrm{CrEuSr}^{\circ}$ \\
\hline 197417 & -19.8 & 1.8 & (4) & 3.6 & 3.0 & 1.2 & $25-10$ & 65 & $*_{\mathrm{AO}} \mathrm{CrEu}$ \\
\hline 199728 & -11.2 & 4.9 & (6) & 12.0 & 2.8 & 4.3 & $<0.1$ & $<30$ & $139 \mathrm{Si}$ \\
\hline 206653 & -11.5 & 6.0 & (3) & 10.3 & 3.6 & 2.9 & $\mathbf{J}$ & $<30$ & B9 Si \\
\hline 207188 & -13.9 & 2.2 & (4) & 4.5 & 3.4 & 1.3 & 25 & 30 & $\mathbf{A 0 ~ S i}$ \\
\hline 208217 & 3.1 & 1.3 & (4) & 2.6 & 3.7 & 0.7 & $>25$ & 55 & *A0 SrCrEu \\
\hline 212385 & 0.2 & 3.6 & (5) & 8.1 & 3.2 & 2.5 & 0.1 & $<30$ & A3 SrCrEu \\
\hline 212432 & 20.3 & 3.1 & (4) & 6.2 & 3.1 & 2.0 & $5-2.5$ & $<30$ & $139 \mathrm{Si}$ \\
\hline 215966 & 25.7 & 3.7 & (4) & 7.3 & 3.2 & 2.3 & $2.5-1$ & $61)$ & 139 EuCr (Sr) \\
\hline 217522 & 34.0 & 2.2 & $(2)$ & 3.1 & 2.2 & 1.4 & - & 45 & A5V CrLuSr (SrSi:) \\
\hline 223967 & 8.5 & 3.7 & (4) & 7.4 & 3.0 & 2.5 & 2.5 .1 & 90 & B39 Si $(139 \mathrm{~V})$ \\
\hline
\end{tabular}




\section{Notes to Table 2:}

HD 3580: Three radial velocities were published by McFadzean et al. (1987). The average is $+5.7 \mathrm{~km} \mathrm{~s}^{-1}$.

HD 27463: Three radial velocities were published by Nordstrom \& Andersen (1985). The average is $+22.3 \pm$ $0.3 \mathrm{~km} \mathrm{~s}^{-1}$.

HD 37808: Three radial velocities were published by Andersen \& Nordstrom (1983). The average is $+20.1 \pm$ $0.9 \mathrm{~km} \mathrm{~s}^{-1}$.

HD 42536: Three radial velocities were published by Nordstrom \& Andersen (1985). It is variable with a range of $58 \mathrm{~km} \mathrm{~s}^{-1}$.

HD 44953: Three radial velocities were published by Andersen \& Nordstrom (1983). The average is $+24.2 \pm$ $0.9 \mathrm{~km} \mathrm{~s}^{-1}$.

HD 61966A: Three radial velocities were published by Andersen \& Nordstrom (1983). The average is $+12.0 \pm$ $1.4 \mathrm{~km} \mathrm{~s}^{-1}$.

HD 80282: There is one radial velocity $\left(+55 \mathrm{~km} \mathrm{~s}^{-1}\right)$ from objective prism published by Donoyelle (1987).

HD 81009: Three radial velocities were published by Nordstrom \& Andersen (1985). The average is $+23.3 \pm$ $0.8 \mathrm{~km} \mathrm{~s}^{-1}$.

HD 83368: Four radial velocities were published by Nordstrom \& Andersen (1985). The average is $-3.2 \pm$ $1.2 \mathrm{~km} \mathrm{~s}^{-1}$.

HD 87653: Donoyelle (1987) published one radial velocity $\left(+4 \mathrm{~km} \mathrm{~s}^{-1}\right)$ from objective prism plates.

HD 90763: Three radial velocities were published by Nordstrom \& Andersen (1985). The average is $-25.7 \pm$ $0.8 \mathrm{~km} \mathrm{~s}^{-1}$.

HD 94660: One radial velocity measurement is in Mathys
(1990): $+25.4 \mathrm{~km} \mathrm{~s}^{-1}$.

HD 101410: Four measurements of the radial were published by Nordstrom \& Andersen (1985). The average is $+0.25 \pm$ $0.2 \mathrm{~km} \mathrm{~s}^{-1}$.

HD 116458: Three radial velocity measurements are available by Nordstrom \& Andersen (1985) and 25 measurements were published by Dworetsky (1982), who computed an orbit classified as class c in Batten et al. (1989). The baricentric radial velocity is $2.5 \mathrm{~km} \mathrm{~s}^{-1}$, and the amplitude $14.7 \mathrm{~km} \mathrm{~s}^{-1}$, with a period of 126.18 days.

HD 128974: Three radial velocities were published by Nordstrom \& Andersen (1985). The average is $-22.9 \pm$ $0.8 \mathrm{~km} \mathrm{~s}^{-1}$.

HD 133792: Three velocities in Nordstrom \& Andersen (1985). The average is $+11.6 \pm 0.7 \mathrm{~km} \mathrm{~s}^{-1}$. Mathys (1990) provided one measure : $+13.2 \mathrm{~km} \mathrm{~s}^{-1}$.

HD 145102: Levato et al. (1987) provided 7 radial velocity measurements with an average of $-1.0 \mathrm{~km} \mathrm{~s}^{-1}$.

HD 147890: Levato et al. (1987) provided 5 radial velocities. The average is $-0.5 \mathrm{~km} \mathrm{~s}^{-1}$.

HD 150846: Zentelis (1983) published one measurement of the radial velocity: $-86.4 \mathrm{~km} / \mathrm{s}$.

HD 151965: There is one measurement $(+8 \mathrm{~km} / \mathrm{s})$ in Perry et al. (1990).

HD 152564: Three measurements in Nordstrom \& Andersen (1985). The average is $-1.4 \pm 1.5$.

HD 155778: Zentelis (1983) published one measurement: -25.6 .

HD 157751: Zentelis (1983) provided one measurement of the radial velocity: $-15.6 \mathrm{~km} / \mathrm{s}$.

HD 199728: Three measurements in Nordstrom \& Andersen (1985). The average is $-10.2 \pm 0.5$. 